\title{
Méthodes d'enquête pour I'estimation des taux démographiques des cheptels de ruminants domestiques tropicaux. Synthèse, limites et perspectives
}

\author{
M. Lesnoff ${ }^{1}$ \\ Article issu de l'atelier Cirad/INRA « Systèmes d'information et outils de pilotage du secteur \\ élevage dans les pays du Sud », Montpellier, France, 11-13 juillet 2011 (cf. l'éditorial)
}

\author{
Mots-clés \\ Ruminant - Bétail - Dynamique \\ des populations - Mortalité - \\ Reproduction - Enquête - \\ Zone tropicale.
}

\begin{abstract}
Résumé
La démographie est un déterminant essentiel de la production des cheptels de ruminants domestiques élevés en conditions extensives tropicales. L'estimation des taux démographiques (taux de reproduction, de mortalité et d'exploitation des animaux) est nécessaire pour évaluer l'effet d'interventions zootechniques sur la production des cheptels. Cette estimation est cependant particulièrement difficile et contraignante. Les systèmes d'élevage traditionnels posent des problèmes spécifiques et importants pour la collecte des données dans les troupeaux. La présente synthèse fait le point sur les méthodes d'enquête utilisées en milieu villageois pour estimer les taux démographiques des ruminants : les suivis individuels d'animaux, les suivis de troupeaux sans identification individuelle des animaux et les enquêtes rétrospectives transversales. Des perspectives de recherche sont proposées pour améliorer les méthodes, notamment dans le cadre des suivis nécessaires pour prendre en compte la variabilité interannuelle des taux démographiques.
\end{abstract}

\section{INTRODUCTION}

Les ruminants domestiques élevés en conditions extensives représentent une valeur économique déterminante pour de nombreux pays du Sud, à l'échelle nationale comme à l'échelle des ménages (74). La démographie animale est un facteur majeur de la production des cheptels de ruminants domestiques (40). Chaque année, le bilan des naissances et des morts au sein des troupeaux se traduit par une production effective (animaux abattus, vendus, etc.) ou une variation du stock animal. La démographie des cheptels dépend de nombreux facteurs incluant le potentiel génétique des animaux, les pratiques d'élevage et les conditions environnementales. Ces facteurs peuvent interagir de manière plus ou moins complexe. Par exemple, une année pluvieuse peut être favorable en termes de disponible alimentaire et donc augmenter la fertilité des femelles reproductrices mais, d'un autre côté, augmenter le risque parasitaire et ainsi défavoriser la survie des animaux. Un autre exemple est qu'une bonne fertilité des femelles en année favorable $n$ pourra se traduire, chez les bovins, par plus de veaux nés en année $n+l$ qui elle sera beaucoup moins favorable. Les modèles

1. Cirad, UMR Systèmes d'élevage méditerranéens et tropicaux, campus international de Baillarguet, TA C-112 / A, F-34398 Montpellier Cedex 5, France. Email : matthieu.lesnoff@cirad.fr démographiques sont particulièrement utiles pour intégrer ces interactions et étudier leurs effets sur la production des cheptels et des troupeaux. Différentes approches de modélisation démographique, mathématiques et informatiques ont été décrites dans la littérature pour diverses zones climatiques (tropicales vs tempérées), aussi bien pour l'élevage extensif qu'intensif. Des typologies de modèles sont régulièrement proposées (par exemple, pour les plus récentes, $1,19,63)$.

Les modèles démographiques sont composés de systèmes d'équations ou de règles informatiques contenant un ensemble de paramètres communément appelés taux démographiques, comme par exemple les taux de reproduction et de mortalité des animaux. Outre leur intérêt comme paramètres des modèles, les taux démographiques sont également considérés comme des indicateurs synthétiques du potentiel zootechnique et des pratiques de conduite des animaux (68). Lorsqu'ils ne sont pas connus par avance, les taux démographiques doivent être estimés à partir de données collectées sur le terrain. Dans les pays du Sud, contrairement aux pays du Nord, l'identification des animaux et la collecte routinière des données animales (entre autres, productions et mobilités géographiques) sont rares. Les zootechniciens travaillant dans ces pays ont été amenés à développer leurs propres dispositifs d'étude de terrain, devant faire face à de nombreuses contraintes. Les troupeaux sont souvent dispersés (divagation des animaux, pâturages 
hors des villages) et mobiles (transhumances saisonnières, nomadisme), et donc difficiles à observer. Les éleveurs ne gardent généralement pas de traces écrites de l'histoire de vie des animaux. Par ailleurs, l'étalement des naissances sur l'ensemble de l'année (malgré des saisonnalités souvent marquées) rend difficile l'identification des différentes cohortes d'animaux présentes dans les troupeaux lors des visites de terrain et, en conséquence, l'estimation de l'âge des animaux. Cette information est cependant essentielle pour l'estimation des taux démographiques.

Après une courte synthèse des connaissances sur la notion de taux démographique, le présent article discute les trois principales méthodes d'enquête utilisées pour estimer les taux démographiques des cheptels de ruminants élevés en conditions extensives dans les zones tropicales : les suivis individuels d'animaux, les suivis de troupeaux sans identification individuelle des animaux, et les enquêtes transversales rétrospectives. Des perspectives de recherche sont également proposées pour améliorer les méthodes d'enquête.

Dans cet article, les méthodes d'enquête sont présentées dans le cadre de l'estimation de taux démographiques moyens à l'échelle de cheptels agrégeant les troupeaux d'un territoire : terroir villageois, région administrative, pays et région climatique. Cette estimation nécessite de réaliser des échantillonnages de troupeaux familiaux, définis ici par les animaux gérés par une même famille. Les techniques d'échantillonnage utilisées, en général basées sur des typologies et des stratifications préliminaires, ne sont pas propres aux taux démographiques et ne font pas l'objet du présent article.

\section{- TAUX DEMOGRAPHIQUES}

De nombreux taux démographiques ont été proposés pour les cheptels domestiques $(9,10,25,30,49,50,54,57)$, cependant souvent sur des bases empiriques. La conception et la mise en œuvre d'une méthode d'enquêtes démographiques nécessite, en préliminaire, de définir précisément quels sont les taux ciblés dans l'étude. Ces taux dépendent eux-mêmes du modèle démographique sous-jacent.

Deux grands types de modèles démographiques sont rencontrés dans la littérature concernant l'élevage tropical $(34,40)$. Le premier type de modèle, le plus simple, décompose le cheptel uniquement par sexe et par classe d'âge $(35,41,67,72)$. Le second type de modèle, plus mécaniste, se distingue au niveau du cheptel femelle. Après une succession de classes d'âge, les femelles atteignent un stade de maturité sexuelle (début des chaleurs), puis le modèle représente explicitement des cycles reproductifs successifs : période de service, fécondation, gestation puis anœstrus postpartum. Après la maturité, l'âge n'est en général pas représenté directement.

Définir le type de modèle utilisé est nécessaire mais non suffisant pour définir les taux démographiques. Il faut également préciser comment sont décomposés les processus démographiques. Par exemple, pour les paramètres des modèles sexe et âge, certains auteurs $(70,75)$ comptabilisent les avortements (c'est-à-dire les gestations non arrivées à terme) et les mort-nés dans la mortalité naturelle, alors que d'autres séparent ces événements. Certains auteurs (76) incluent également dans la mortalité naturelle les abattages d'urgence, c'est-à-dire les abattages effectués lorsque l'animal ne peut plus se déplacer en raison d'un accident ou d'une maladie, alors que d'autres les considèrent comme des exploitations car la viande de ces animaux peut en général être valorisée. Ce type de variantes existe également pour la reproduction.
Certains auteurs (66) incluent notamment les avortements et les mort-nés dans le taux de mise bas, alors que d'autres définissent des taux spécifiques.

Les décompositions choisies pour les processus démographiques doivent être clairement exprimées au niveau des questionnaires d'enquête car, au final, ces choix ont des effets élevés sur la valeur des estimations. Par exemple, dans des troupeaux bovins au Kenya (32), un tiers de la mortalité rapportée pour les veaux entre 0 et 1 an provenait des avortements et des mort-nés. Dans des troupeaux de petits ruminants au Mali (70), 5 des 28 p. 100 de mortalité rapportée pour les jeunes entre 0 et 5 mois provenaient d'avortements.

Enfin, la conception ou le choix d'une méthode d'enquête peuvent être guidés par la définition mathématique des taux démographiques ciblés. Un taux démographique peut représenter deux paramètres mathématiques distincts, une probabilité vs un taux instantané (en anglais hazard rate), qui ont des formules de calcul et des interprétations différentes $(40,44,45)$. Certaines méthodes d'enquête sont plus ou moins bien adaptées à l'estimation de l'un ou de l'autre paramètre.

Dans le présent article, les méthodes d'enquêtes démographiques sont discutées dans le contexte des modèles démographiques sexe et âge. Ces modèles contiennent deux types de taux démographiques $(44,45)$ : les taux naturels qui quantifient des performances animales (reproduction et mortalité naturelle des animaux) et les taux de gestion qui réfèrent à des événements liés directement à des décisions de l'éleveur (abattage, vente, achat, prêt et don d'animaux). Ces derniers peuvent être décomposés en taux d'exploitation (sorties d'animaux des troupeaux) et en taux d'importation (entrées d'animaux dans les troupeaux). La figure 1 montre un exemple de décomposition des processus démographiques utilisée récemment pour les enquêtes en milieu tropical $(45,46)$, et les taux démographiques correspondants sont listés dans le tableau I. D'autres décompositions ont été proposées par le passé $(26,49)$.



Figure 1 : schéma des événements démographiques dans les troupeaux utilisé pour définir la liste des paramètres démographiques présentés dans le tableau $I$. Source : Lesnoff et coll., 2007, Quae, adapté de Landais et Sissokho, 1986, Cirad-lemvt. 


\section{Tableau I}

Taux démographiques utilisés comme paramètres d'entrées dans les modèles démographiques simulant la dynamique d'un cheptel par sexe et classe d'âge. Les taux sont définis par période de temps et catégorie d'animaux

\begin{tabular}{|c|c|}
\hline Taux naturel & Définition \\
\hline (1) Taux de mise bas & Probabilité ou taux instantané a qu'une femelle ait une mise bas \\
\hline (2) Taux d'avortement ${ }^{b}$ & Probabilité ou taux instantané qu'une femelle ait un avortement \\
\hline (3) Taux de prolificité & Nb. moyen de produits (nés vivants + mort-nés) par mise bas \\
\hline (4) Taux de mortinatalité ${ }^{c}$ & Probabilité qu'un produit soit mort-né \\
\hline (5) Taux de femelles à la naissance & Probabilité qu'un produit né vivant soit une femelle \\
\hline (6) Taux de mort naturelle ${ }^{d}$ & $\begin{array}{l}\text { Probabilité ou taux instantané qu'un animal meure de mort naturelle durant une période } \\
\text { de temps donnée }\end{array}$ \\
\hline Taux de gestion & Définition \\
\hline (7) Taux d'exploitation & Probabilité ou taux instantané qu'un animal soit exploité (abattage e , vente, prêt, don, etc.) \\
\hline (8) Taux d'importation & Probabilité ou taux instantané qu'un animal soit importé dans le troupeau (achat, prêt, don, etc.) \\
\hline
\end{tabular}

Sources : Lesnoff et coll., 2007, Quae, 2011, Quae

${ }^{a}$ Un taux instantané correspond à ce qui est appelé hazard rate en anglais. Les différences conceptuelles entre taux instantannés et probabilités et les formules de calcul sont détaillées dans Lesnoff et coll., 2007, Quae, 2011, Quae.

${ }^{\mathrm{b}}$ Un avortement est une gestation qui n'a pas été à son terme et qui a engendré un produit non viable.

${ }^{\mathrm{c}}$ Les mort-nés ne sont pas inclus dans la mortalité. La mortalité ne concerne que les produits nés vivants.

${ }^{\mathrm{d}}$ La mortalité naturelle correspond à toutes les causes de mort excépté les abattages. Les abattages d'urgence en raison d'accidents ou de maladies sont considérés comme des exploitations et non des mortalités.

e A l'intérieur de la ferme.

\section{SUIVIS INDIVIDUELS}

\section{Principes}

Le suivi individuel des animaux est la méthode de référence pour collecter des informations sur les performances zootechniques (17, 29, 62, 69), notamment les données servant à l'estimation des taux démographiques. La méthode consiste à suivre pendant une ou plusieurs années un même échantillon de troupeaux dont tout ou partie des animaux sont identifiés individuellement, le plus souvent par des boucles auriculaires. Des enquêteurs spécialement formés visitent régulièrement les troupeaux, par exemple tous les quinze jours ou tous les mois. A chaque visite, ils font l'inventaire des animaux présents et notent tous les événements démographiques survenus depuis la visite précédente (mise bas, mortalité, exploitation et importation d'animaux). D'autres données peuvent également être collectées au niveau individuel (par exemple, croissance pondérale, état nutritionnel, production laitière) ou par groupe d'animaux (alimentation, soins vétérinaires). Chaque événement démographique est référencé par sa date d'occurrence, ce qui permet ensuite de calculer avec fiabilité les taux démographiques par période de temps et par catégorie d'animaux.

\section{Applications}

En Afrique subsaharienne, les suivis individuels ont été mis en œuvre dans plusieurs contextes. Avant les années 1980, la majorité des études étaient réalisées dans des stations expérimentales dans lesquelles les conditions d'élevage étaient souvent « améliorées » (par exemple par l'utilisation systématique d'intrants alimentaires ou vétérinaires). L'alimentation des animaux était plus ou moins contrôlée (pâturage et complémentation) et tous les animaux recevaient les prophylaxies et les traitements vétérinaires nécessaires à leur développement. Un des objectifs était d'évaluer le potentiel des races locales ou des animaux issus de croisements avec des races exotiques.

Les zootechniciens se sont ensuite intéressés à des suivis individuels en dehors du milieu contrôlé, en réalisant des suivis directement auprès des éleveurs. Bien que plus difficiles à mener, ces dispositifs répondaient à une volonté de mieux connaître les performances des troupeaux dans les conditions représentatives de l'élevage traditionnel $(7,17,33,77)$. Ces performances sont en général bien inférieures à certaines atteintes dans les stations de recherche. Par exemple en Afrique de l'Ouest, des suivis individuels de ruminants ont été mis en place entre 1980 et 1995 au Niger $(21,22)$, au Mali $(73,77)$, au Sénégal $(18,24)$ et en Gambie $(2,58,79)$.

Ces études novatrices ont permis d'améliorer considérablement les connaissances sur la productivité des troupeaux et les pratiques d'exploitation des animaux en élevage traditionnel. Plusieurs institutions internationales ont eu un rôle moteur dans cette démarche comme le Centre de coopération internationale en recherche agronomique pour le développement (Cirad), l'International Livestock Research Institute (ILRI, anciennement International Livestock Centre for Africa ou ILCA) et l'International Trypanotolerance Centre (ITC). Deux suivis particulièrement longs ont été mis en place et maintenus au Sénégal par le Cirad en collaboration avec l'Institut sénégalais de recherche agricole (ISRA) : un suivi de treize années (1983-95) des petits ruminants (projet PPR « Pathologies et productivité des petits ruminants ») et un suivi de neuf années (1990-98) sur des bovins (projet ABT «Alimentation du bétail »).

\section{Systèmes d'information}

Les suivis individuels génèrent des données répétées sur le même animal. Ceci nécessite une organisation particulière des questionnaires et des bases informatiques pour la gestion et la valorisation 
des données. Un système d'information gratuit et disponible pour les suivis individuels dans les élevages extensifs tropicaux est le logiciel Laser (http://livtools.cirad.fr; 27). Ce système d'information résulte d'une série de travaux de calibration des suivis réalisés sur le terrain, dont les premiers ont été effectués au nord de la Côte d'Ivoire dans les années 1970 (62), puis finalisés au Sénégal au début des années 1980. Une première version du système d'information a abouti au logiciel Panurge (17), fonctionnant sous MSDOS et utilisé jusqu'à la fin des années 1990 dans de nombreux pays (par exemple, Burkina Faso, Côte d'Ivoire, Nouvelle-Calédonie, Sénégal). Profitant d'une évolution importante des langages informatiques et d'un nouveau travail conceptuel sur l'organisation des données collectées (28), le système d'information a été redéveloppé en Visual Basics et utilise une base de données MS Access. Il peut gérer des données de différentes natures, notamment démographiques (reproduction, mortalité, exploitation), pathologiques (symptôme, sérologie), sur la production (lait, poids vif, état corporel, entre autres), sur l'insémination artificielle et relatives à des interventions zootechniques sur les animaux. Le calcul des taux démographiques à partir de suivis individuels nécessite de nombreuses manipulations de données, souvent difficiles du fait de la nature et de la diversité des données, notamment les mesures répétées sur le même animal et le référencement des événements par des dates. Pour Laser, des routines automatiques de calcul ont été développées $(44,45)$ et regroupées dans un package de fonctions programmées avec le logiciel statistique gratuit $\mathrm{R}$ (www.r-project. org ; 64).

D'autres systèmes d'information de suivis individuels sont disponibles mais sous forme de logiciels commerciaux payants ou initialement développés pour les élevages intensifs des pays du Nord. Certains ont cependant été utilisés pour l'élevage extensif tropical. Par exemple, l'ILRI a utilisé le système Vampp Dairy Management, un logiciel développé au Canada (www.vampp.com) pour des suivis dans des systèmes d'élevage laitier au Kenya et en Ouganda. Le système InterHerd (www.interagri.org) développé au : Royaume-Uni a été utilisé dans des projets de recherche menés par เn l'Université de Wageningen dans les pays du Sud. Enfin, le système BeefPro (www.beefpro.net) développé en Afrique du Sud par l'Agricultural Research Council (ARC) est très utilisé en Afrique australe pour la gestion de ranchs d'élevage bovin.

\section{- SUIVIS DE TROUPEAUX SANS IDENTIFICATION DES ANIMAUX}

Les suivis de troupeaux sans identification individuelle des animaux, appelés « suivis de troupeaux » dans la suite de l'article, sont une simplification des suivis individuels (30). Comme ces derniers, ils sont basés sur des visites régulières des troupeaux, par exemple par quinzaine, mois ou trimestre. Lors de chaque visite, l'enquêteur fait l'inventaire des animaux présents dans le troupeau, puis des événements démographiques survenus depuis la dernière visite. Cependant, les informations collectées ne sont plus référencées par numéro d'animal ni liées entre les visites : l'enquêteur n'est pas tenu de reconnaître les animaux d'une visite à l'autre. Ceci facilite la collecte de données et allège le travail de terrain qui peut être réalisé par des agents spécialement formés mais aussi par des éleveurs.

Bien que plus légers, les suivis de troupeaux présentent des difficultés méthodologiques (que nous aborderons ci-dessous dans la section discussion) qui peuvent expliquer leur utilisation encore limitée sur le terrain $(3,5,23,51)$, ainsi que l'absence d'outils génériques (questionnaires standards, systèmes d'information et routines automatiques de calcul) pour ces méthodes.

\section{ENQUETES RETROSPECTIVES}

\section{Principes}

Les enquêtes rétrospectives sont transversales, c'est-à-dire que les troupeaux ne sont enquêtés qu'une fois et tous à la même période de l'année (idéalement le même mois pour que les informations entre troupeaux soient comparables). Elles ont été utilisées depuis de nombreuses années en Afrique. Des premiers documents méthodologiques ont été publiés dès 1975 (66) et ces méthodes ont probablement été utilisées depuis plus longtemps (10).

$\mathrm{Au}$ cours de la visite, l'enquêteur reconstitue, d'après la déclaration de l'éleveur, les événements démographiques survenus dans le troupeau à plus ou moins long terme. Deux principales méthodes sont utilisées : la méthode des carrières des femelles (ou progeny history method, ou encore mature breeding female history method) et la méthode des douze derniers mois.

La méthode des carrières $(10,13,66)$ semble la plus ancienne et a probablement été adaptée de techniques d'enquête sur les lignées familiales en démographie humaine. Différents protocoles sont possibles. Par exemple, dans un troupeau, il s'agit tout d'abord de reconstituer la carrière reproductrice complète d'un sous-échantillon de femelles présentes (en général cinq ou six femelles si la taille du troupeau le permet). L'enquêteur note les mises bas successives depuis la naissance jusqu'au jour de l'enquête et les âges respectifs des femelles lors des mises bas. Les femelles du sous-échantillon doivent être nées dans le troupeau (l'éleveur doit connaître leur histoire) et être suffisamment âgées pour avoir déjà mis bas. Ensuite, pour chacune des mises bas déclarées, il s'agit de décrire le devenir de chaque jeune produit : l'enquêteur demande à l'éleveur si le jeune est encore présent dans le troupeau à la date de l'enquête, sinon la cause de son absence (par exemple, mort naturelle, abattage ou vente) et l'âge de la sortie.

La méthode des carrières est censée estimer des taux démographiques représentant des moyennes sur une période rétrospective pluriannuelle pouvant s'étendre de trois à dix ans avant la date de l'enquête, selon la durée des carrières des femelles sélectionnées. Les taux de mise bas sont estimés d'après les mises bas déclarées pour les différentes femelles, et les taux de mortalité et d'exploitation d'après le devenir des jeunes issus des mises bas.

La méthode des douze derniers mois (25) fournit des taux uniquement pour l'année précédant l'enquête. Sur le terrain, la méthode consiste à faire l'inventaire des animaux présents dans le troupeau au moment de l'enquête et à en estimer l'âge, puis à noter tous les événements démographiques (mise bas, mortalité, exploitation et importation) survenus par sexe et classe d'âge dans le troupeau lors des douze derniers mois.

Bien que toutes les deux basées sur le déclaratif de l'éleveur, les méthodes des carrières et des douze derniers mois sont différentes des enquêtes rétrospectives appelées participatives (ou participative rural appraisals ou PRA ; 8). Les méthodes démographiques sont quantitatives et beaucoup de données sont collectées au niveau de l'animal, par exemple lors de l'inventaire des événements démographiques. Les PRA sont basées sur des entretiens auprès de groupes d'éleveurs (par exemple des comités villageois) fournissant des réponses collectives souvent semi-quantitatives ou qualitatives. L'analyse des données PRA nécessite ensuite la mise en œuvre de techniques d'ordination.

\section{Applications}

Les enquêtes rétrospectives ont été principalement utilisées comme des outils de diagnostic rapide pour dégager les grandes 
caractéristiques démographiques de cheptels, à destination d'institutions de recherche ou de décideurs cherchant à donner des priorités dans les actions à mener (49). Depuis la fin des années 1980 , les institutions de recherche ont été régulièrement sollicitées par les services techniques de l'élevage des pays du Sud pour réaliser des évaluations nationales de la démographie des cheptels de ruminants. Par exemple, des enquêtes carrière nationales ont été menées au Tchad, au Cameroun et en République centrafricaine $(11,56,61)$. Plus récemment (2007), une enquête utilisant la méthode des douze derniers mois a été menée par les services de l'élevage du Niger. Ce type d'étude se situe davantage dans le domaine de l'expertise que de la recherche. L'objectif attendu pour les pays est de disposer d'estimations moyennes de la productivité et de l'exploitation du cheptel pour faciliter l'élaboration des rapports statistiques annuels sur les productions animales nationales. En pratique, l'atteinte de cet objectif s'avère cependant très difficile (38).

Plus rarement, les enquêtes rétrospectives sont utilisées pour des objectifs de recherche, aussi bien la méthode des carrières $(12$, $15,52)$ que celle des douze derniers mois $(5,48)$. Par exemple en 2010, des enquêtes douze derniers mois ont été menées dans quatre pays d'Afrique de l'Ouest (Gambie, Guinée, Mali et Sénégal) dans le cadre d'un projet sur la gestion durable des races locales de ruminants (www.progebe.net). Cependant, la majorité des résultats d'enquêtes rétrospectives est publiée sous forme de littérature grise (53). Ceci est principalement dû à la forte incertitude attachée aux données collectées (cf. la section « Avantages et limites des méthodes »).

\section{Systèmes d'information}

Comme pour les suivis de troupeaux, la mise en œuvre des méthodes rétrospectives souffre d'un manque de formalisation et d'outils génériques mis à disposition des utilisateurs. La méthode des douze derniers mois a cependant fait l'objet d'une formalisation récente. Des travaux menés dans plusieurs projets de recherche financés par la Coopération belge (DGCD au Niger) et la Commission européenne (Niger, Burkina Faso) entre 2006 et 2010 ont permis d'élaborer l'outil 12MO (http://livtools.cirad.fr ; $39,46,47)$ en collaboration avec différents instituts de recherche (Cirad, ILRI, Icrisat, Agrhymet) et services techniques (ministères de l'élevage du Niger et du Burkina Faso). La méthode 12MO propose une chaîne complète allant du protocole de terrain au calcul des paramètres démographiques. L'outil comprend un protocole d'enquête et deux questionnaires standards (Q1 et Q2) devant être remplis pour chaque troupeau enquêté : Q1 Inventaire et description des animaux présents et, pour les femelles, reproduction des femelles lors des douze derniers mois, et Q2 Entrées et sorties lors des douze derniers mois. Une base de données au format MS Access et une interface permettent les saisies et la gestion des données. Un package de fonctions développé avec le logiciel $\mathrm{R}$ (www.r-project.org) permet le calcul automatisé des taux démographiques à partir de la base de données. Cet outil $12 \mathrm{MO}$ a déjà été utilisé dans plusieurs pays d'Afrique subsaharienne, au niveau de terroirs villageois, de points d'eau pastoraux et à l'échelle nationale.

\section{AVANTAGES ET LIMITES DES METHODES}

\section{Suivis individuels}

Les suivis individuels apportent des données précises et fiables, en raison des inventaires réguliers des animaux et des événements démographiques au sein des troupeaux mais aussi de la relation de confiance qui s'établit avec l'éleveur. Les données étant collectées par date, les taux démographiques peuvent être estimés à n'importe quelle échelle de temps, par exemple par quinzaine ou par mois pour les variations saisonnières ou par année pour les variations interannuelles $(36,43)$. Les suivis individuels sont recommandés pour établir des référentiels techniques sur la productivité des troupeaux ou, dans des démarches d'expérimentation, pour estimer l'effet et le coût/bénéfice d'interventions diverses (entre autres, vaccination, déparasitage, complémentation). Ils ont cependant plusieurs limites. Ils sont assez lourds à mettre en place et à maintenir dans la durée. La constitution de l'échantillon des troupeaux suivis demande souvent de nombreuses négociations préliminaires avec les éleveurs. Il faut ensuite inventorier et boucler tous les animaux des troupeaux de l'échantillon et mettre en place le système de recueil des informations. Cette étape est particulièrement laborieuse pour les superviseurs des enquêtes et les enquêteurs. Souvent coûteux en moyens humains et financiers, les suivis individuels peuvent difficilement être étendus à de grandes régions ou à l'échelle d'un pays. A notre connaissance, seul le projet PPR sur les petits ruminants au Sénégal $(18,69)$ a réussi à appréhender la diversité des taux démographiques à l'échelle nationale, en mettant en place des suivis dans trois sites géographiques répartis suivant un gradient agroclimatique nord-sud.

Les suivis individuels posent également des difficultés lorsque les troupeaux sont très mobiles, notamment dans le cas des éleveurs nomades. Les troupeaux se dispersent dans les zones pastorales sans accès routier ni plan de route prédéterminé. Il peut devenir alors pratiquement impossible de les localiser et d'effectuer des visites suffisamment régulières, même si cette contrainte doit être modulée en raison du développement du téléphone mobile et de la bonne couverture réseau dans certains pays. Les suivis individuels réalisés sur des élevages réellement nomades ont ainsi été très rares. Un tel suivi a été réalisé sur des troupeaux camelins au Niger (59) avec beaucoup de difficultés pratiques (M. Saley, ministère des Ressources animales du Niger, commun. pers.). Les transhumances saisonnières observées dans les milieux agropastoraux posent moins de difficultés (73) car les informations individuelles sur les animaux peuvent en général être reconstruites au retour des troupeaux avec des biais acceptables (et, dans les cas favorables de transhumances de faible amplitude, les enquêteurs peuvent contacter les éleveurs et visiter les troupeaux sur les lieux de transhumance).

Enfin, les suivis individuels ne peuvent répondre à l'évaluation $a$ posteriori de l'impact de phénomènes imprévisibles comme les chocs climatiques ou épidémiques, sauf dans le cas particulier où le choc survient en cours du suivi.

\section{Suivis de troupeaux}

Les suivis de troupeaux se heurtent à beaucoup de contraintes rencontrées dans les suivis individuels, notamment pour les systèmes d'élevage nomade. Ils sont cependant plus légers à mettre en place sur le terrain et à pérenniser car les enquêteurs n'ont pas à marquer les animaux en début de suivi, ni à les identifier individuellement lors des visites. En contrepartie, la méthode est soumise à deux difficultés méthodologiques.

La première difficulté est l'estimation de l'âge des animaux présents dans le troupeau, qui doit être effectuée à chaque visite (ce que n'a pas à faire l'enquêteur dans un suivi individuel car, les animaux étant identifiés, la date de naissance de chaque animal est connue et peut être utilisée pour calculer l'âge de l'animal à tout moment). L'âge des animaux est estimé à dire d'éleveur, ce qui crée des biais dans la structure par âge du troupeau et donc, ultérieurement, dans les estimations des taux démographiques par classe d'âge. Par ailleurs, cette étape peut devenir coûteuse en 
temps d'enquête avec pour conséquence une possible lassitude de l'éleveur (à chaque visite, l'enquêteur doit requestionner l'éleveur sur les âges des animaux).

La seconde difficulté concerne l'enquête des troupeaux pratiquant des transhumances saisonnières. Pendant ces transhumances qui peuvent durer de deux à six mois, voire plus, le troupeau peut disparaître totalement de la zone d'enquête. Dans ce cas, lorsque les animaux sont identifiés individuellement, l'enquêteur peut essayer, au retour du troupeau, de reconstituer et dater tous les événements démographiques survenus depuis le début de la transhumance. Pratiquement aucune information n'est perdue. Mais pour les suivis de troupeaux, ce travail de reconstitution est beaucoup plus difficile. L'élaboration de questionnaires à la fois simples et permettant une estimation correcte des taux démographiques à partir de suivis de troupeaux transhumants est une question encore non résolue.

\section{Enquêtes rétrospectives}

De durée en général inférieure à deux mois, les enquêtes rétrospectives sont plus légères que les suivis et peuvent être réalisées plus facilement sur le terrain, notamment lors de stages d'étudiants. $\mathrm{Ne}$ demandant qu'une seule visite par troupeau, elles peuvent s'appliquer à tous les systèmes d'élevage, y compris les éleveurs nomades enquêtés au niveau de points de concentration, comme les campements ou les points d'eau. Elles peuvent également être étendues à de grandes zones, jusqu'à l'échelle nationale. Enfin, des enquêtes peuvent être lancées suffisamment rapidement pour quantifier les pertes animales, suite à l'occurrence de chocs inattendus (notamment avec la méthode des douze derniers mois).

La limite principale des enquêtes rétrospectives est qu'elles fournissent des résultats beaucoup plus incertains que les suivis. Elles sont en effet fortement soumises à des biais d'enquête engendrés par les défauts de mémoire (oublis d'événements démographiques, biais sur les âges estimés, etc.) ou les fausses déclarations des éleveurs, mais aussi à des biais de calcul provenant de la manière dont sont agrégées les données dans les enquêtes $(37,39)$.

La méthode la plus sensible au biais d'enquête est la méthode des carrières. Lors de l'entretien, l'éleveur doit remonter loin dans l'histoire de la femelle et de sa progéniture pour se remémorer les mises bas et le devenir des jeunes produits (par exemple, jusqu'à dix ans ou plus pour la carrière d'une vache). Si les réponses peuvent être fiables pour les systèmes nomades dans lesquels les éleveurs vivent en permanence avec les troupeaux, elles sont souvent moins fiables pour d'autres systèmes utilisant des bergers temporaires. Dans les régions agricoles, par exemple, il existe une rotation assez rapide des bergers et la personne enquêtée est souvent dans l'impossibilité de connaître l'histoire complète des femelles présentes dans le troupeau. Certains biais d'enquête peuvent être partiellement évités en limitant le nombre d'enquêteurs, ce qui permet une meilleure formation avant les enquêtes et une supervision plus poussée sur le terrain. Ceci est cependant difficile dans les études de grande envergure, avec des équipes d'enquêteurs de formation et de qualité hétérogène. En général, les biais sont loin d'être négligeables, surtout pour les taux de mortalité et d'exploitation estimés d'après le devenir des jeunes produits. Par exemple, une enquête carrière réalisée au Niger (12) rapporte pour les petits ruminants sahéliens des taux de mortalité et d'exploitation (tous âges confondus) respectivement de 1-2 et 8 p. 100. Ces estimations sont très éloignées des valeurs de référence (respectivement $>10$ et $>30$ p. 100), connues par ailleurs pour ces espèces en zone sahélienne. La sous-estimation des taux de mortalité et d'exploitation semble être une caractéristique récurrente de la méthode des carrières $(20,56,60)$.
La méthode des douze derniers mois est moins soumise aux biais d'enquête car l'effort de mémoire demandé à l'éleveur est moins important. Cependant, la méthode peut être sensible aux formules d'estimations utilisées pour calculer les taux démographiques, notamment pour les petits ruminants (problèmes non détaillés ici ; 39,46 ). Elle est également sensible à la date choisie pour l'enquête en raison de la saisonnalité des événements démographiques (43). Enfin, une limite importante de la méthode est que les taux estimés ne concernent que l'année précédant l'enquête, excepté pour le taux de mise bas dont une estimation sur le plus long terme peut être obtenue d'après la corrélation entre l'âge et la parité des femelles, collectés lors de l'inventaire des animaux du troupeau (46). Les taux démographiques des cheptels tropicaux étant très variables d'une année à l'autre, les estimations obtenues d'après une enquête transversale sur les douze derniers mois ne sont en général pas conformes aux performances moyennes des troupeaux sur le long terme.

\section{- PERSPECTIVES METHODOLOGIQUES}

\section{Conception des méthodes d'enquête}

Beaucoup de travaux restent à mener pour concevoir et améliorer les méthodes d'enquêtes démographiques en milieu tropical. Il semble utopique d'envisager à court terme un système pérenne d'identification généralisée des animaux dans les pays, même si des initiatives spécifiques pourraient émerger pour certains types d'élevages comme les éleveurs laitiers périurbains (certains utilisent déjà des cahiers de suivis individuels). Les zootechniciens seront donc confrontés pour longtemps à la nécessité de mettre en place des dispositifs d'enquête sur le terrain pour obtenir des données fiables. Les méthodes finalisées et documentées sont encore trop peu nombreuses, aussi bien au niveau des protocoles (questionnaires) que des systèmes d'information (bases de données et interfaces de saisie) disponibles. Cela peut entraîner des démarches empiriques sur le terrain qui desservent la qualité des données. Améliorer la standardisation des méthodes et leur documentation permettrait aux utilisateurs d'avoir une meilleure connaissance des résultats attendus et au final un meilleur usage des enquêtes démographiques.

\section{Précision statistique des estimations}

L'estimation des taux démographiques dans une zone d'étude nécessite l'échantillonnage de troupeaux. Cet échantillonnage crée une incertitude statistique dont il faut connaître l'étendue (précision des estimations). En élevage tropical, comme pour les pays du Nord, l'échantillonnage des troupeaux peut s'appuyer sur les techniques classiques de tirage aléatoire, notamment l'échantillonnage stratifié et par degré (le degré pouvant être notamment un district, un village ou un troupeau). Une caractéristique du milieu tropical est cependant l'absence de base d'échantillonnage qui entraîne deux difficultés : la réalisation des tirages aléatoires et l'extrapolation à l'échelle du territoire des taux moyens estimés au niveau de l'échantillon. Ces deux points sont fortement associés aux questions d'inventaire des troupeaux et des animaux dans les territoires, nécessitant elles-mêmes de nombreux travaux de recherche et dépassant le cadre de cet article.

Quelle que soit la méthode d'échantillonnage, il est nécessaire de connaître l'effet de la taille des échantillons (nombre de troupeaux et d'animaux enquêtés) sur la précision des estimations des taux démographiques. A notre connaissance, cette question n'a pas été étudiée de manière systématique dans le contexte de l'élevage tropical, malgré son importance pour effectuer des recommandations lors de la mise en place des dispositifs d'enquête sur le terrain. 
Les modèles statistiques utilisés pour estimer des probabilités $p$ ou des taux instantanés $h$ sont en général des modèles linéaires généralisés. L'hypothèse usuelle de ces modèles est que les données suivent une distribution binomiale pour $p$ et une distribution de Poisson pour $h$. Ces distributions supposent l'indépendance des animaux présents dans l'échantillon.

Dans les enquêtes démographiques, cependant, cette hypothèse est en général invalide. Les animaux sont échantillonnés par degré, et ces degrés peuvent créer des corrélations entre animaux échantillonnés. Par exemple, en raison de facteurs externes divers, les animaux d'un même troupeau sont en général plus semblables (par rapport à la variable étudiée) que les animaux de troupeaux différents. Dans ce cas, l'échantillonnage des animaux par troupeau entraîne des corrélations intra-troupeaux qui créent une « surdispersion » des données par rapport à l'hypothèse d'indépendance $(14,55,65)$. Cette surdispersion a pour conséquence de diminuer l'efficacité statistique des échantillonnages : il faut alors augmenter le nombre de troupeaux et d'animaux échantillonnés pour avoir une précision statistique acceptable.

Dans le cadre des méthodes discutées dans cette synthèse, l'étude de cette efficacité statistique des plans d'échantillonnage pourrait être réfléchie en deux étapes. Il s'agirait tout d'abord de rassembler un ensemble suffisamment large de bases de données démographiques sur l'élevage tropical (par exemple, celles disponibles dans les institutions internationales), puis de les utiliser pour estimer les gammes de variation des moyennes des taux démographiques et des paramètres de surdispersion. Sur la base des résultats obtenus, il s'agirait ensuite à l'aide de modèles statistiques adaptés aux données surdispersées de simuler aléatoirement des données démographiques et d'estimer les variations de précision des estimations en fonction de la taille des échantillons $(16,78)$.

\section{Besoins en suivis}

Une difficulté majeure pour la recherche en élevage tropical, outre la capitalisation et la valorisation insuffisantes des données collectées sur les performances animales $(31,34)$, est le manque d'information quantitative longitudinale. La compréhension et la modélisation des interactions entre les facteurs environnementaux (climat, maladies, etc.), les ressources en pâturage et les performances zootechniques des troupeaux nécessitent par exemple de disposer de séries temporelles permettant d'estimer les variations saisonnières et interannuelles des paramètres de production animale, dont les taux démographiques. Ces données sont aujourd'hui quasi inexistantes pour les systèmes d'élevage traditionnels tropicaux.

Malgré leur grand intérêt, les dispositifs de suivis tels que ceux mis en place en Afrique de l'Ouest (Sénégal, Mali) au milieu des années 1980 n'ont pas été renouvelés. Les institutions scientifiques et les bailleurs de fonds ont globalement orienté les efforts de recherche vers l'élaboration de modèles conceptuels, mathématiques ou informatiques de plus en plus performants et complexes, dont les paramètres sont fixés à dires d'expert ou estimés d'après des enquêtes légères. La plupart des études sur les taux démographiques menées dans les systèmes d'élevage tropicaux depuis 15 ans ont ainsi été réalisées à partir d'enquêtes transversales rétrospectives ou participatives, fournissant des données peu fiables et constituant souvent des photographies ne tenant pas compte de la variabilité interannuelle. Cette tendance a été renforcée à la fin des années 1990 en pensant que l'apport de nouvelles technologies, comme les modèles mathématiques et informatiques, données satellitaires, accès aux bases de données internationales sur le web, allait permettre de limiter massivement les besoins en collecte de données sur le terrain.
Aujourd'hui, le niveau d'incertitude sur la dynamique et la productivité des troupeaux des pays du Sud montre que cette démarche n'a pas atteint ses objectifs. Elle a plutôt limité nos capacités actuelles de diagnostic et de prospective sur les systèmes d'élevage. Par exemple, une analyse de sensibilité sur la dynamique post-sécheresse des cheptels bovins sahéliens (41) a récemment montré que le taux de mise bas des vaches était un paramètre clé de la résilience démographique des troupeaux après des pertes animales importantes. Un taux de mise bas de $0,45 /$ vache/an ou de $0,65 /$ vache/an modifie fortement la compréhension que l'on peut avoir de la capacité du cheptel à se reconstituer. Or, les données disponibles au Sahel sont insuffisantes pour privilégier l'une des deux valeurs, en dehors d'une appréciation à dire d'expert. Dans des régions plus humides, deux autres études récentes ont abouti aux mêmes conclusions, avec des taux de mise bas de $0,34 / v a c h e /$ an en Ethiopie (42) et de 0,51/vache/an au Mali (4). Dans les deux cas, les données ont été collectées lors de suivis de courte durée (18 mois en Ethiopie et 12 mois au Mali). Face à la forte variabilité interannuelle du taux de mise bas et en l'absence de résultats moyens à long terme disponibles dans la littérature, il n'a pas été possible de déterminer si ces estimations étaient conformes aux performances moyennes des troupeaux dans ces zones ou si elles correspondaient à des extrêmes. Une dernière illustration concerne l'impact sur les productions animales des systèmes d'élevage d'Afrique de l'Ouest, des actions sanitaires menées par les Etats depuis quinze ans (contrôle des maladies) ou des plans de recommandation sur les pratiques d'alimentation des troupeaux. Les hypothèses avancées sont souvent optimistes mais aucune donnée précise et objective n'est aujourd'hui disponible pour les valider ou les infirmer.

Il paraît ainsi essentiel pour la recherche agronomique en zone tropicale de relancer des dispositifs pérennes permettant d'établir de nouveaux référentiels biotechniques sur les troupeaux dans les systèmes d'élevage traditionnels. Les institutions de recherches internationales ou occidentales travaillant dans les pays du Sud ont une responsabilité importante et un rôle majeur à jouer dans ce défi futur. Diverses voies peuvent favoriser la mise en place de dispositifs longitudinaux. Tout d'abord, il est important de convaincre les décideurs et les bailleurs de fond de l'intérêt de ces dispositifs. Ceci peut être notamment réalisé en montrant les limites qu'engendre sur nos modèles prospectifs l'incertitude existant autour des taux démographiques, dans le même esprit que l'illustration proposée pour la dynamique post-sécheresse d'un cheptel (41). Ensuite, il convient d'intégrer davantage les enquêtes démographiques dans des dispositifs observatoires associant d'autres disciplines, notamment l'épidémiologie, l'économie, l'agronomie et la sociologie. Enfin, il semble capital de proposer des innovations qui permettent d'alléger les collectes de données longitudinales sur le terrain. Ceci nécessite de mener des recherches méthodologiques. Sans être exhaustif, cette synthèse propose dans les lignes qui suivent des pistes pour identifier certaines de ces innovations.

\section{Durée des suivis}

Rares sont les projets de recherche qui pourront maintenir en continu des suivis de plus de dix années comme celui du projet PPR au Sénégal (18). La question se pose alors de la durée minimale des suivis pour l'estimation des taux démographiques, par exemple pour établir des référentiels biotechniques. L'effet de cette durée sur le biais et la précision des estimations dépend de la variabilité interannuelle des taux démographiques, qui dépend elle-même des données environnementales et des systèmes d'élevage considérés. Des études simulant aléatoirement des sous-échantillonnages d'années à partir de bases de données longitudinales existantes (par exemple les données PPR et ABT) 
pourraient apporter des premiers éléments de réponse et des recommandations.

\section{Simplification des suivis individuels}

\section{Sous-échantillonnage d'animaux dans les troupeaux}

Dans le protocole usuel des suivis individuels, tous les animaux d'un troupeau échantillonné sont identifiés et suivis, y compris les animaux nés ou importés dans le troupeau au cours du suivi. $\mathrm{Ce}$ suivi exhaustif est coûteux en temps lors des visites d'élevages. Le travail peut être allégé si seulement quelques animaux par troupeau (quatre ou cinq) sont identifiés et suivis. Ceci permettrait, par exemple, d'augmenter l'effectif de troupeaux échantillonnés, ce qui est particulièrement intéressant pour estimer des variabilités entre troupeaux ou entre zones géographiques.

L'échantillonnage des animaux au sein des troupeaux pose cependant des questions méthodologiques. Parmi d'autres, comment échantillonner les animaux suivis au sein du troupeau (par exemple, de manière aléatoire, de manière raisonnée en suivant des quotas par rapport à des structures sexe-âge estimées par ailleurs), combien échantillonner d'animaux par troupeau (effets sur la précision des estimations), comment renouveler l'échantillon pour remplacer les animaux suivis sortis du troupeau (morts, vendus, etc.) ou comment estimer les taux d'importation avec un tel protocole sont des questions ouvertes. Comme pour l'effet de la durée d'enquête sur les estimations, certaines des questions posées peuvent être explorées en simulant aléatoirement des sous-échantillonnages d'animaux à partir de bases de données de suivis individuels existantes.

\section{Identification des animaux}

Les suivis individuels mis en place en Afrique subsaharienne ont pratiquement tous nécessité la pose de boucles auriculaires numérotées. Ce système est efficace et robuste mais a des limitations opérationnelles. Les enquêteurs sont obligés de lire les boucles sur chaque animal (qu'il faut immobiliser) et de les retranscrire sur leurs fiches d'enquête. Ces numéros sont ensuite enregistrés dans la base de données centralisant les informations par des opérateurs de saisie. Toute cette chaîne d'opérations est coûteuse en temps et engendre des erreurs de transcription. Ces erreurs sont détectées automatiquement dans la base de données par des routines informatiques mais leur correction est laborieuse (il faut renvoyer une fiche de correction sur le terrain). Un autre problème est la perte des boucles. Les animaux concernés doivent être rebouclés, ce qui implique des changements de numéros de boucle, source d'erreurs supplémentaires. Enfin, l'identification par boucle auriculaire ne fournit aucune information sur la mobilité géographique des troupeaux et des animaux, à moins de mettre en place un protocole lourd utilisant des enquêteurs itinérants (71).

Une alternative au bouclage auriculaire est l'identification électronique, notamment à l'aide d'un bolus intestinal (6). Placé dans le tube digestif des animaux par voie orale, le bolus est sans danger et peu de rejets sont observés (6). La puce électronique contenue dans le bolus peut stocker différentes informations sur l'animal, comme l'identification et l'état physiologique, et peut être lue électroniquement à l'aide d'un récepteur manuel placé quelques secondes à côté de l'animal ou de pistolets viseurs. Cette technologie est de plus en plus utilisée en Europe. Pour les élevages extensifs tropicaux, elle permettrait de faciliter la collecte des données sur le terrain lors des visites de suivis individuels.

Son opérationnalité dans des milieux où les animaux sont soumis à des stress récurrents (maladie, sous-alimentation, déplacement pour le pâturage ou abreuvement) reste cependant à évaluer et il est nécessaire dans ce contexte de planifier des tests en conditions villageoises. Par ailleurs, les protocoles de transfert des données du bolus vers les systèmes d'information existants sont à standardiser avant de pouvoir diffuser ces technologies auprès d'utilisateurs comme les institutions de recherche et les organisations non gouvernementales.

\section{Alternatives aux suivis individuels}

S'ils sont la référence, les suivis individuels peuvent être des opérations lourdes. Comme indiqué précédemment, les suivis de troupeaux (sans identification des animaux) peuvent être une bonne alternative aux suivis individuels, mais un travail de standardisation des méthodes est à mener. Une autre alternative serait de remplacer les suivis par la réplication d'enquêtes rétrospectives au cours du temps.

La méthode des douze derniers mois pourrait notamment être répliquée chaque année à la même période, sur un même échantillon de troupeaux ou sur un échantillon partiellement ou totalement renouvelé chaque année. L'outil 12MO (46) présenté dans cet article semble par exemple intéressant pour estimer la variabilité interannuelle des taux démographiques.

Bien que les méthodes rétrospectives soient très souvent utilisées au détriment des suivis pour des raisons de coût, leur réplication longitudinale n'a cependant pas encore été testée en Afrique subsaharienne. La pertinence de l'approche nécessite par ailleurs une meilleure validation des méthodes rétrospectives. Quelques études ont discuté des biais théoriques de la méthode des douze derniers mois $(37,39)$ mais les biais d'enquêtes issus des déclarations des éleveurs n'ont jamais été estimés. Ceci limite la capacité d'interprétation des résultats de ce type d'enquête. Des protocoles de validation peuvent être proposés. Il s'agirait par exemple de mettre en place des suivis sur trois ou quatre ans (individuels ou de troupeaux) servant de référence et, au cours de ces suivis, de mener des enquêtes rétrospectives ponctuelles sur les troupeaux (ou d'autres troupeaux similaires : même zone et mêmes caractéristiques) pour enfin comparer les estimations obtenues avec les deux méthodes.

\section{- CONCLUSION}

L'estimation des taux démographiques (reproduction, mortalité et exploitation des animaux) est nécessaire pour évaluer les productions issues des cheptels tropicaux, mais elle est particulièrement difficile et contraignante. Les systèmes d'élevage traditionnels posent des problèmes spécifiques pour la collecte des données démographiques au sein des troupeaux. Il n'existe malheureusement pas de méthode miracle. Face à la variabilité interannuelle élevée des performances démographiques, il est important de favoriser les suivis pluriannuels. Les suivis individuels, méthode de référence, sont recommandés lorsqu'ils peuvent être mis en œuvre. Les méthodes rétrospectives sont plus légères mais fournissent des résultats pouvant être biaisés, qui doivent être utilisés avec précaution. Les taux démographiques des systèmes d'élevage mobiles sont particulièrement difficiles à évaluer en raison des coûts liés à la mobilité des animaux. Pour les systèmes d'élevage sédentaires, les suivis de troupeaux (sans identification des animaux) paraissent être un bon intermédiaire entre les suivis individuels et les enquêtes rétrospectives, mais la méthode souffre d'un manque d'outils standardisés. Il est important de mener des recherches méthodologiques pour améliorer l'ensemble de ces méthodes d'enquête. 


\section{Remerciements}

Ce travail a été mené partiellement dans le cadre du projet «Elevage, climat et société » (Eclis) financé par l'Agence nationale de la recherche (ANR, France). L'auteur remercie les quatre lecteurs anonymes dont les commentaires ont permis d'améliorer significativement cet article.

\section{BIBLIOGRAPHIE}

1. AGABRIEL J., INGRAND S., 2004. Modelling the performance of the beef cow to build a herd functioning simulator. Anim. Res., 53: 347361.

2. ANKERS P., ITTY P., ZINSSTAG J., TRAWALLY S., PFISTER K., 1998. Biannual anthelmintic treatments in village Djallonke sheep in the Gambia: effects on productivity and profitability. Prev. Vet. Med., 34: 215-225.

3. AWA D.N., NJOYA A., NGOTAMA A.C., 2000. Economics of prophylaxis against peste des petits ruminants and gastrointestinal helminthosis in small ruminants in North Cameroun. Trop. Anim. Health Prod., 32: 391-403.

4. BA A., LESNOFF M., MOULIN C.H., 2011. Demographic dynamics and off-take of cattle herds in south Mali. Trop. Anim. Health Prod., 43: 1101-1109.

5. BEBE B.O., UDO H.M.J., ROWLANDS G.J., THORPE W., 2003. Smallholder dairy systems in the Kenya highlands: cattle population dynamics under increasing intensification. Livest. Prod. Sci., 82: 211221.

6. CAJA G., GHIRARDI J.J., HERNANDEZ-JOYER M., MILAN M.J., BOCQUIER F., 2006. Use of electronic boluses for the traceability of ruminants: state of the art, implementation and evaluation in sheep and cattle. In : Rencontres Recherches Ruminants, Paris, France, 8-9 déc. 2006, p. 163-166.

7. CAMUS E., LANDAIS E., POIVEY J.P., 1981. Genetic structure of sedentary cattle in the North of Ivory Coast. Future prospects of cattle production following the spread of zebu crossing. Rev. Elev. Méd. Vét. Trop., 34: 187-198. [in French with English abstract]

8. CATLEY A., ALDERS R.G., WOOD J.L.N., 2012. Participatory epidemiology: Approaches, methods, experiences. Vet. J., 191: 151160.

9. CIRAD-IEMVT, 1972. Définition des paramètres zootechniques concernant la reproduction, l'économie des troupeaux, l'économie du bétail de boucherie en élevage bovin, ovin, caprin. Maisons-Alfort, France, Cirad-lemvt.

10. CIRAD-IEMVT, 1989. Les enquêtes sur la productivité du bétail. Maisons-Alfort, France, Cirad-lemvt. (Fiche technique $n^{\circ} 5$ )

11. CIRAD-IEMVT, 1988. Résultats de l'enquête sur la situation de l'élevage bovin, ovin et caprin au Tchad. Maisons-Alfort, France, Cirad-lemvt.

12. COLIN DE VERDIERE P., 1994. Etude comparée de trois systèmes agro-pastoraux dans la région de Filingue (Niger). Les conséquences de la sédentarisation de l'élevage pastoral au Sahel. Stuttgart, Allemagne, Université d'Hohenheim / Maisons-Alfort, France, CiradEMVT.

13. DESTA L., COPPOCK D.L., 2002. Cattle population dynamics in the southern Ethiopian rangelands, 1980-97. J. Range Manage., 55: 439-451.

14. DIGGLE P.J., LIANG K.Y., ZEGER S.L., 1994. Analysis of longitudinal data. Oxford, UK, Oxford Science.

15. DUMAS R., 1980. Contribution to the study of small ruminants in Chad. Rev. Elev. Méd. Vét. Trop., 33: 215-233. [in French with English abstract]

16. DURAN PACHECO G., HATTENDORF J., COLFORD JR J.M., MAUSEZAHL D., SMITH T., 2009. Performance of analytical methods for overdispersed counts in cluster randomized trials: Sample size, degree of clustering and imbalance. Stat. Med., 28: 2989-3011.

17. FAUGERE O., FAUGERE B., 1986. Flock monitoring and control of individual performances of small ruminants bred in an African traditional environment. Methodology features. Rev. Elev. Méd. Vét. Trop., 39: 29-40. [in French with English abstract]
18. FAUGERE O., MERLIN P., FAUGERE B., 1991. Méthodologie d'évaluation de la santé et de la productivité des petits ruminants en Afrique : I'exemple du Sénégal. Rev. Sci. Tech. Off. Int. Epizoot., 10 : 103-130.

19. GOUTTENOIRE L., COURNUT S., INGRAND S., 2011. Modelling as a tool to redesign livestock farming systems: a literature review. Animal, 5: 1957-1971.

20. HASSAN W.A., 2000. Biological productivity of sheep and goats under agrosilvo-pastoral systems in the Zamfara Reserve in north-western Nigeria. Göttingen, Germany, Cuvillier Verlag.

21. HAUMESSER J.B., 1975. Some aspects of reproduction in the reddish brown goats of Maradi. Comparison with other tropical or sub-tropical breeds. Rev. Elev. Méd. Vét. Trop., 28: 225-234. [in French with English abstract]

22. HAUMESSER J.B., GERBALDI P., 1980. Husbandry and breeding ability of Nigerian Uda sheep. Rev. Elev. Méd. Vét. Trop., 33: 205-213. [in French with English abstract]

23. HUTTNER K., LEIDL K., PFEIFFER D., KASAMBARA D., JERE F.B.D., 2001. The effect of a community-based animal health service programme on livestock mortality, off-take and selected husbandry applications. A field study in northern Malawi. Livest. Prod. Sci., 72: 263-278.

24. ICKOWICZ A., MBAYE M., 2001. Sudanian forest and cattle feeding in Senegal: potential and limits. Bois For. Trop., 270: 47-61.

25. ILCA, 1990. Livestock systems research manual. Working Paper 1, Vol. 1. Addis Ababa, Ethiopia, ILCA.

26. ILRI, 2000. Handbook of livestock statistics for developing countries. Socio-economics and Policy Research Working Paper 26. Nairobi, Kenya, ILRI.

27. JUANES X., LANCELOT R., 1999. LASER : logiciel d'aide au suivi d'élevages de ruminants. Montpellier, France, Cirad.

28. LANCELOT R., FAYE B., JUANES X., NDIAYE M., PEROCHON L., TILLARD E., 1998. The Baobab database: a tool for modeling small ruminants production and health in traditional farming systems in Senegal. Rev. Elev. Méd. Vét. Trop., 51: 135-146. [in French with English abstract]

29. LANDAIS E., FAUGERE O., 1986. Un modèle illustré de système d'investigation pour l'étude pluridisciplinaire des systèmes d'élevage en milieu traditionnel. Cah. Rech. Dév., 25 : 75-94.

30. LANDAIS E., SISSOKHO M.M., 1986. Bases méthodologiques du contrôle des performances animales pour l'analyse zootechnique et démographique : collecte des données et choix des variables. Méthodes pour la recherche sur les systèmes d'élevage en Afrique intertropicale. Maisons-Alfort, France, Cirad-lemvt, p. 433-485. (Etudes et synthèses n 20)

31. DE LEEUW P.N., MCDERMOTT J.J., LEBBIE S.H.B., 1995. Monitoring of livestock health and production in sub-Saharan Africa. Prev. Vet. Med., 25: 195-212.

32. DE LEEUW P.N., SEMENYE P.P., PEACOCK C.P., GRANDIN B.E. 1991. Productivity of cattle and smallstock. In: Bekure S., De Leeuw P.N., Grandin B.E., Neate P.J.H., eds., Maasai herding. An analysis of the livestock production system of Masai pastoralists in eastern Kajiado District, Kenya. Addis Ababa, Ethiopia, ILCA, p. 127-140.

33. DE LEEUW P.N., WILSON R.T., 1987. Comparative productivity of indigenous cattle under traditional management in sub-Saharan Africa. Q. J. Int. Agric., 26: 377-390.

34. LESNOFF M., 2011. Démographie et zootechnie tropicales : un lien par les modèles matriciels appliqués aux cheptels de ruminants dans les élevages extensifs. Mém. Habilitation à diriger des recherches, Université Montpellier II / Cirad, Montpellier, France.

35. LESNOFF M., 1999. Dynamics of a sheep population in a Sahelian area (Ndiagne district in Senegal): A periodic matrix model. Agric. Syst., 61: 207-221.

36. LESNOFF M., 2000. Etude de la dynamique et de la productivité des populations domestiques tropicales par les modèles matriciels en temps discret: les populations d'ovins au Sénégal. Thèse Doct., Université Montpellier II, Montpellier, France, $270 \mathrm{p}$.

37. LESNOFF M., 2008. Evaluation of 12-month interval methods for estimating animal-times at risk in a traditional African livestock farming system. Prev. Vet. Med., 85: 9-16.

38. LESNOFF M., 2009. Projet ENST. Renforcement des capacités opérationnelles du MRA sur les enquêtes en élevage. Montpellier, France, Cirad. 
39. LESNOFF M., 2009. Reliability of a 12-month period retrospective survey method for estimating parturition and mortality rates in a traditional African livestock farming system. Rev. Elev. Méd. Vét. Trop. 62: 49-57.

40. LESNOFF M., 2013. Simulating dynamics and productions of tropical livestock populations - mmage: An R package for discrete time matrix models. Montpellier, France, CIRAD, http://livtools.cirad.fr.

41. LESNOFF M., CORNIAUX C., HIERNAUX P., 2012. Sensitivity analysis of the recovery dynamics of a cattle population following drought in the Sahel region. Ecol. Model., 232: 28-39.

42. LESNOFF M., DIEDHIOU M., LAVAL G., BONNET P. WORKALEMAHU A., KIFLE D., 2002. Demographic parameters of a domestic cattle in a contagious-bovine-pleuropneumonia infected area of Ethiopian highlands. Rev. Elev. Méd. Vét. Trop., 55: 139-147.

43. LESNOFF M., LANCELOT R., 2009. Evaluation of crude annual parturition rate estimates in a small-holder African ruminant farming system. Animal, 3: 1347-1353.

44. LESNOFF M., LANCELOT R., MOULIN C.H., 2007. Calcul des taux démographiques dans les cheptels de ruminants domestiques tropicaux approche en temps discret. Montpellier, France, Quae, www.quae.com.

45. LESNOFF M., LANCELOT R., MOULIN C.-H., MESSAD S., JUANES X., SAHUT C., 2011. Calculation of demographic parameters in tropical livestock herds - A discrete time approach with LASER animal-based monitoring data. Montpellier, France, Quae, www.quae.com.

46. LESNOFF M., MESSAD S., JUANES X., 2010. 12MO: A crosssectional retrospective method for estimating livestock demographic parameters in tropical small-holder farming systems. Montpellier, France, CIRAD, http://livtools.cirad.fr.

47. LESNOFF M., SALEY M., ADAMOU K., N'DJAFA OUAGA $\mathrm{H}$. AYANTUNDE A., GERARD B., 2008. 12MO: A retrospective method for estimating demographic parameters in tropical ruminant livestock populations. Montpellier, France, CIRAD.

48. LESNOFF M., SALEY M., ADAMOU K., N'DJAFFA H., 2007. Enquête démographique 2006 sur le cheptel domestique au Niger: sites du Fakara, de Gabi et de Zermou. Rapport préliminaire. Nairobi, Kenya, ILRI.

49. LHOSTE P., DOLLE V., ROUSSEAU J., SOLTNER D., 1993. Manuel de zootechnie des régions chaudes - Les systèmes d'élevage. Paris, France, ministère de la Coopération. (Coll. Précis d'élevage)

50. LIENARD G., LEGENDRE J., 1974. Productivité en veaux des troupeaux de vaches allaitantes. Bull. Tech. Centre Rech. Zootech. Vét. Theix ( $\mathrm{n}^{\circ}$ spécial) : 47-67

51. MADANI T., HUBERT B., VISSAC B., CASABIANCA F., 2002 Analysis of cattle husbandry and farming systems changes in an Algerian sylvopastoral area. Rev. Elev. Méd. Vét. Trop., 55: 197-209. [in French with English abstract]

52. MARICHATOU H., MAMANE L., BANOIN M., BARIL G., 2002. Zootechnical performances of goats in Niger: Comparative study of the Maradi Russet goat with the Black-Coat goat in Maradi area. Rev. Elev. Méd. Vét. Trop., 55: 79-84. [in French with English abstract]

53. MARSHALL K., EJLERTSEN M., POOLE J., 2011. Sustainable management of globally significant endemic ruminant livestock in West Africa (PROBGEBE): Estimate of livestock demographic parameters in the Gambia. Nairobi, Kenya, ILRI. (Research Report 28)

54. MARZIN J., LIENARD G., 1984. Productivité en agneaux des troupeaux ovins. Réflexions sur le choix d'une méthode d'analyse. Bull. Tech. Centre Rech. Zootech. Vét. Theix, 56: 69-90.

55. MCCULLOCH C.E., SEARLE S.R., NEUHAUS J.M., 2008 Generalized, linear, and mixed models. New York, USA, Wiley, 424 p.

56. MEYER C., ROMIER G., LESNOFF M., LE MASSON A., MESSAD S., FAYE B., 1997. Enquête sur l'élevage du bétail de République centrafricaine. Montpellier, France, Cirad-EMVT.

57. MUKASA-MUGERWA E., 1989. A review of reproductive performance of female Bos indicus (zebu) cattle. Addis Ababa, Ethiopia, ILCA. (Monograph No 6)

58. OSAER S., GOOSSENS B., JEFFCOATE I., HOLMES P., 1998. Effects of Trypanosoma congolense and nutritional supplements in Djallonke ewes on live weight during pregnancy, post partum weight, haematology parameters and lamb performance. Res. Vet. Sci., 80: 215-230.
59. PACHOLEK X., LANCELOT R., LESNOFF M., MESSAD S., 2000. Growth performance of camel calves raised in the pastoral zone of Niger. Rev. Elev. Méd. Vét. Trop., 53: 189-197 [in French with English abstract]

60. PEACOCK C.P., 1983. A rapid appraisal of goat and sheep flock demography in East and West Africa: method, results and application to livestock research and development. Addis Ababa, Ethiopia, ILCA. (Working Document 28)

61. PLANCHENAULT D., 1992. Enquête sur la productivité du bétail camerounais. Rapport final. Maisons-Alfort, France, Cirad-lemvt.

62. POIVEY J.P., SEITZ J.L., LANDAIS E., 1981. Purposes and methodological aspects of a computerized individual monitoring system applied to small holders' cattle in the North of Ivory Coast. Rev. Elev. Méd. Vét. Trop., 34: 199-210. [in French with English abstract]

63. PUILLET L., SAUVANT D., TICHIT M., 2010. Intérêts et limites de la prise en compte de la variabilité individuelle dans les modèles de fonctionnement du troupeau. Prod. Anim., 23: 255-268.

64. R DEVELOPMENT CORE TEAM, 2011. R: A language and environment for statistical computing. Vienna, Austria, R Foundation for Statistical Computing.

65. SEARLE S.R., CASELLA G., MCCULLOCH C.E., 1992. Variance components. New York, USA, Wiley.

66. SEDES, 1975. Etude de la structure et de la dynamique des troupeaux bovins : méthodologie pratique. Paris, France, ministère de la Coopération.

67. TACHER G., 1975. Notes sur la démographie bovine au Sahel. I. Représentation et projection théorique du troupeau par un modèle mathématique. Rev. Elev. Med. Vet. Trop., 28: 547-569.

68. TICHIT M., INGRAND S., MOULIN C.H., COURNUT S., LASSEUR J., DEDIEU B., 2004. Analyse de la diversité des trajectoires productives des femelles reproductrices : intérêt pour la modélisation du fonctionnement du troupeau en élevage allaitant. Prod. Anim., 17: 123132.

69. TILLARD E., MOULIN C.H., FAUGERE O., FAUGERE B., 1997. Le suivi individuel des petits ruminants au Sénégal : un mode d'étude des troupeaux en milieu villageois. Prod. Anim., 10: 67-78.

70. TRAORE A., WILSON R.T., 1988. Livestock production in Central Mali: environmental and pathological factors affecting morbidity and mortality of ruminants in the agro-pastoral system. Prev. Vet. Med., 6: 6375

71. TURNER M.D., HIERNIAUX P., 2002. The use of herders' accounts to map livestock activities accross agropastoral landscape in semi-arid Africa. Land. Ecol., 17: 367-385.

72. UPTON M., 1993. Livestock productivity assessment and modelling. Agric. Syst., 43: 459-472.

73. WAGENAAR K.T., DIALLO A., SAYERS A.R., 1986. Productivity of transhumant Fulani cattle in the inner Niger delta of Mali. Ababa, Ethiopia, ILCA. (Research Report 13)

74. WILSON R.T., 1989. Livestock production in central Mali: economic characters and productivity indices for Sudanese Fulani cattle in the agro-pastoral system. Trop. Agric. (Trinidad), 66: 49-53.

75. WILSON R.T., 1988. Small ruminant production systems in tropical Africa. Small Rumin. Res., 1: 305-325.

76. WILSON R.T., CLARKE S.E., 1976. Studies on the livestock of Southern Darfur, Sudan. II: Production traits in cattle. Trop. Anim. Health Prod., 8: 47-51.

77. WILSON R.T., LIGHT D., 1986. Livestock production in central Mali: economic characters and productivity indices for traditionnally managed goats and sheep. J. Anim. Sci., 62: 567-575.

78. ZHANG H., LU N., FENG C., THURSTON S.W., XIA Y., ZHU L., TU X.M., 2011. On fitting generalized linear mixed-effects models for binary responses using different statistical packages. Stat. Med., 30: 2562-2572.

79. ZINSSTAG J., ANKERS P., ITTY P., NJIE M., KAUFMANN J., PANDEY V.S., PFISTER K., 1997. Effect of strategic gastrointestinal nematode control on fertility and mortality of N'Dama cattle in the Gambia. Vet. Par., 73: 105-117.

Accepté le 14.10.2013 


\section{Summary}

Lesnoff M. Survey methods to estimate demographic rates of tropical ruminant livestock herds. Review, limits and prospects

Demography is a key determinant of the production of ruminant livestock herds raised under extensive tropical conditions. Estimation of demographic rates (reproduction, mortality and offtake) is necessary to assess the impacts of husbandry or health interventions on herd production. This estimation is however particularly difficult and constraining. Traditional livestock systems pose major specific problems when attempting to collect data from herds. This paper reviews survey methods used in villages to estimate demographic rates of ruminants, i.e. individual animal-based monitoring, herd monitoring without individual identification of animals, and retrospective cross-sectional surveys. Research perspectives are proposed to improve methods, especially in the context of longitudinal surveys that include interannual variability of demographic rates.

Keywords: Ruminant - Livestock - Population dynamics Mortality - Reproduction - Survey - Tropical zone.

\section{Resumen}

Lesnoff $\mathbf{M}$. Métodos de encuesta para la estimación de tasas demográficas en hatos de ganado rumiante. Síntesis, límites y prospectos

La demografía es un determinante clave de la producción de hatos de ganado rumiante criado bajo condiciones tropicales extensivas. La estimación de tasas demográficas (reproducción, mortalidad y consumo) es necesaria para asesorar los impactos de la ganadería o de las intervenciones sanitarias sobre la producción del hato. Sin embargo, esta estimación es particularmente difícil y restrictiva. Los sistemas de ganadería tradicionales presentan importantes problemas específicos durante los intentos de recolección de información en los hatos. El presente artículo revisa los métodos de encuesta utilizados en los pueblos para estimar las tasas demográficas de los rumiantes, por ejemplo, seguimiento basado en el animal individual, seguimiento de hato sin identificación individual de los animales y encuestas transversales retrospectivas. Se proponen perspectivas de investigación para mejorar los métodos, especialmente en el contexto de encuestas individuales, incluyendo la variabilidad entre años de las tasas demográficas.

Palabras clave: Rumiante - Ganado - Dinámica de poblaciones - Mortalidad - Reproducción - Encuesta - Zona tropical. 
